\title{
DIHYDROMYRICETIN ATTENUATES HIGH GLUCOSE-INDUCED CASPASE 3 EXPRESSION, ROS PRODUCTION AND INCREASES AMPK PHOSPHONYLATION IN PC12 CELLS
}

\author{
Shuo Wang ${ }^{1 \&}$, Lin Wang ${ }^{1 \&}$, Haijian $\mathrm{Li}^{1 \&}$, Shumei Wang ${ }^{1 \&}$, Zhenzhen $\mathrm{Li}^{1}$, Bao $\mathrm{Li}^{\mathbf{2}^{*}}$, Chunzhen $\mathrm{Zhao}^{{ }^{*}}$ \\ ${ }^{1}$ Laboratory of Applied Pharmacology, Weifang Medical University, Weifang 261053, China \\ ${ }^{2}$ Department of urology, Affiliated Hospital of Weifang Medical College
}

Article Info: Received 17 August 2019; Accepted 28 October. 2019

DOI: https://doi.org/10.32553/jbpr.v8i5.674

Co-first authors ${ }^{8}$ : These authors contributed equally to the work.

Corresponding author: Chunzhen Zhao, Li Bao

Conflict of interest statement: No conflict of interest

\section{ABSTRACT:}

Dihydromyricetin (DMY) has a protective effect on neural function under central nervous system dysfunction conditions. There is growing interest concerning the beneficial effects of DMY on treating diabetic neuropathy (DN). This study was carried to detect protective effects of DMY on high glucose (HG)-induced cell damage and related mechanisms. The effect of DMY on cell survival was detected by MTT assay. Caspase-3 and phosphorylated AMP-activated protein kinase (AMPK) was evaluated by Western blotting. The effects of DMY and AMPK agonist AICAR on ROS production was determined. Our results showed that DMY treatment protect against HG-induced cell damage. DMY treatment significantly reduced the expression of caspase-3 and phosphorylated AMPK. ROS production was inhibited by DMY or AMPK agonist AICAR treatment. These studies demonstrate that DMY may inhibit ROS production, caspase-3 expression through AMPK pathway.

Keywords: dihydromyricetin, caspase, oxidative stress

\section{Introduction}

Diabetes mellitus is a major cause of high morbidity and mortality in the world. Diabetic neuropathy (DN) is one of the most common diabetes-related complications leading to cognitive impairment, motor and sensory dysfunction ${ }^{[1]}$. The importance of DN is being recognized increasingly. Oxidative stress, impaired cerebral insulin signaling systems are considered as being closely related to DN development ${ }^{[2]}$. Many evidence have reported that overproduction of reactive oxygen species (ROS) contributed to neural cell apoptosis and inhibition of oxidation may block the progression of $\mathrm{DN}^{[3]}$.

Dihydromyricetin (DMY), a flavonoid compound, was isolated from the leaves of Ampelopsis grossedentata $^{[4]}$. It has been reported to possess multiple pharmacological activities including antiinflammatory ${ }^{[5]}$, anti-oxidative ${ }^{[6]}$, anticancer ${ }^{[7]}$ and hepatoprotective effects ${ }^{[8]}$. Recent data supported dihydromyricetin attenuated methylglyoxal induced- oxidative stress in PC12 cells $^{[9]}$.
Dihydromyricetin treatment can improve $A \beta$ induced cognitive impairment and reverse progressive neuropathology in a mouse model of Alzheimer's disease ${ }^{[10]}$. However, the effects of DMY on hyperglycemia neural injury and related mechanisms have not been clearly studied.

The aim of the present study was to investigate the protective effects of DMY on high glucose-induced cell damage in cultured PC12 cells. We further examined whether DMY can affect high glucoseinduced caspase-3 expression, ROS production and AMPK phosphonylation.

\section{Materials and Methods}

\section{Reagents}

Dihydromyricetin (purity $\geq 98 \%$ ) was purchased from Sigma-Aldrich. ROS production was measured by using a ROS assay kit (Nanjing Jiancheng Bioengineering Institute, China). Antibodies against phospho-AMPK, cleaved caspase 3 was purchased from Boster Bio Tech (Wuhan, China). Antibodies 
against GAPDH were obtained from Beyotime, Nanjing, China.

\section{Cell Culture}

PC12 cells were cultured in DMEM medium containing $10 \%$ fetal bovine serum under conditions of $37^{\circ} \mathrm{C}$ in humidified air containing 5\% $\mathrm{CO}_{2}$. The cultured medium was changed every 48 hours and the cells in the exponential phase of growth were used in all experiments.

\section{Cell viability detection}

Cell viability was evaluated by a modified 3-(4,5dimethylthiazol-2-yl)-2,5-diphenyltetrazolium bromide (MTT) detection assay. PC12 cells were seeded in 96-well plates. Cells were pretreated with different conditions of Dihydromyricetin (10, 20, $40 \mu \mathrm{M}$ ) for $1 \mathrm{~h}$ and treated with high glucose with concentration of $100 \mathrm{mM}$ for $48 \mathrm{~h}$. MTT (5 $\mathrm{mg} / \mathrm{mL}$ ) was added to each well, and the cells were incubated for $4 \mathrm{~h}$ at incubator of $37{ }^{\circ} \mathrm{C}$. The medium was removed and dimethyl sulfoxide (DMSO) was added to the wells. The absorbance was measured at $570 \mathrm{~nm}$ using multiscan spectrum. Cell viability was expressed normalized to percentage of non-treated control cells.

\section{Intracellular ROS detection assay}

Intracellular ROS production was evaluated by 2,7dichlorofluorescin diacetate (DCFH-DA) fluorescent probe detection assay. PC12 cells were seeded in 24-well plates and pretreated with DMY $20 \mu \mathrm{M}$ for $1 \mathrm{~h}$ or AICAR $10 \mathrm{nM}$ and exposed to $100 \mathrm{mM}$ of HG for $48 \mathrm{~h}$. The medium was removed and incubated with $20 \mu \mathrm{mol} / \mathrm{L}$ of DCFH-DA for $30 \mathrm{~min}$ at $37{ }^{\circ} \mathrm{C}$, then cells were washed with PBS to remove DCFHDA. Cells were collected and analyzed immediately using a microplate reader.

\section{Western Blot Analysis}

PC12 cells were harvested and washed with icecold PBS, and the cellular lysates were prepared. Protein concentrations were determined by BCA assay. The protein samples were subjected to electrophoresis on $10-12 \%$ SDS polyacrylamide gels and transferred onto a nitrocellulose membrane. Then the membrane was blocked with $7.5 \%$ nonfat milk, and incubated with anti-cleaved caspase 3, anti- phospho-AMPK and anti-GAPDH antibodies overnight.
Then the blots were incubated with anti-rabbit IRDye700DX ${ }^{\circledR}$-conjugated antibody or anti-mouse IRDye800DX ${ }^{\circledast}$-conjugated antibody. The signals were scanned by an Odyssey infrared imaging system. Protein bands were quantitatively evaluated by Quantity One ${ }^{\circledR}$ analysis software. The ratio to confidence reference items was calculated.

\section{Statistical Analysis}

All data were expressed as mean \pm SD. The significance of differences among three or more groups was performed using One-way ANOVA followed by Bonferroni's post hoc test (SPSS 15.0 for Windows, SPSS inc., USA). $P<0.05$ was considered statistically significant.

\section{Results}

\section{DMY attenuated HG-induced PC12 cell damage}

To investigate protective effect of DMY in high glucose-induced neural cell damage model, PC12 cells were pretreated with DMY with different concentrations of $10,20,40 \mu \mathrm{M}$ and exposed to high glucose with concentrations of $100 \mathrm{mM}$ for 48 h. Then cell viability was detected by MTT detection assay. HG induced cell damage significantly compared to the control group. DMY with concentrations of $10,20,40 \mu \mathrm{M}$ can increase cell survival significantly as compared to HG group (Fig.1, $P<0.01$ ).

\section{DMY decreased HG-induced caspase-3 expression in PC12 cells}

Apoptosis related protein caspase 3 was detected by Western blotting and protective effect of DMY on caspase-3 expression was evaluated. As shown in Fig.2, compared to control group, cleaved caspase-3 protein expression increased significantly. $20 \mu \mathrm{M}$ of DMY can significantly reduced caspase- 3 expression in PC12 cells compared to HG group (Fig.2A and 2B, $P<0.05$ ).

\section{Effect of DMY on HG-induced intracellular ROS production and AMPK activation}

Many studies have reported that ROS contributed to HG-induced PC12 cell injury. Here we struggled to demonstrate whether DMY would affect the elevated level of ROS and related mechanisms in HG-treated PC12 cells. Our results showed that DMY significantly reduced HG-induced ROS production in PC12 cells. Also we found DMY can recover HG-induced inhibition of AMPK 
phosphonylation (Fig.2, $P<0.05$ ). As compared with HG group, AMPK agonist AICAR $10 \mathrm{nM}$ can significantly reduced HG-induced ROS level in PC12 cells (Fig.3, $P<0.01$ ).

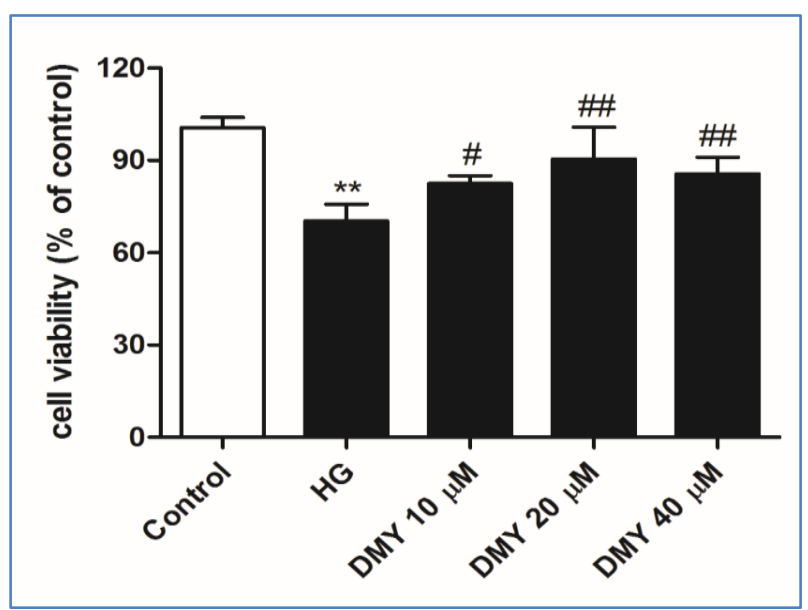

Fig.1: Effect of DMY in PC12 cells after HG exposure. Cell viability was evaluated by MTT detection assay. PC12 cells are pretreated with DMY for $1 \mathrm{~h}$ and then exposed to HG $100 \mathrm{mM}$ for $48 \mathrm{~h}$. Data are expressed as mean \pm SD. ${ }^{* *} P<0.01$ compared to control group. ${ }^{\# \#} P<0.01,{ }^{\#} P<0.05$ compared to HG group.

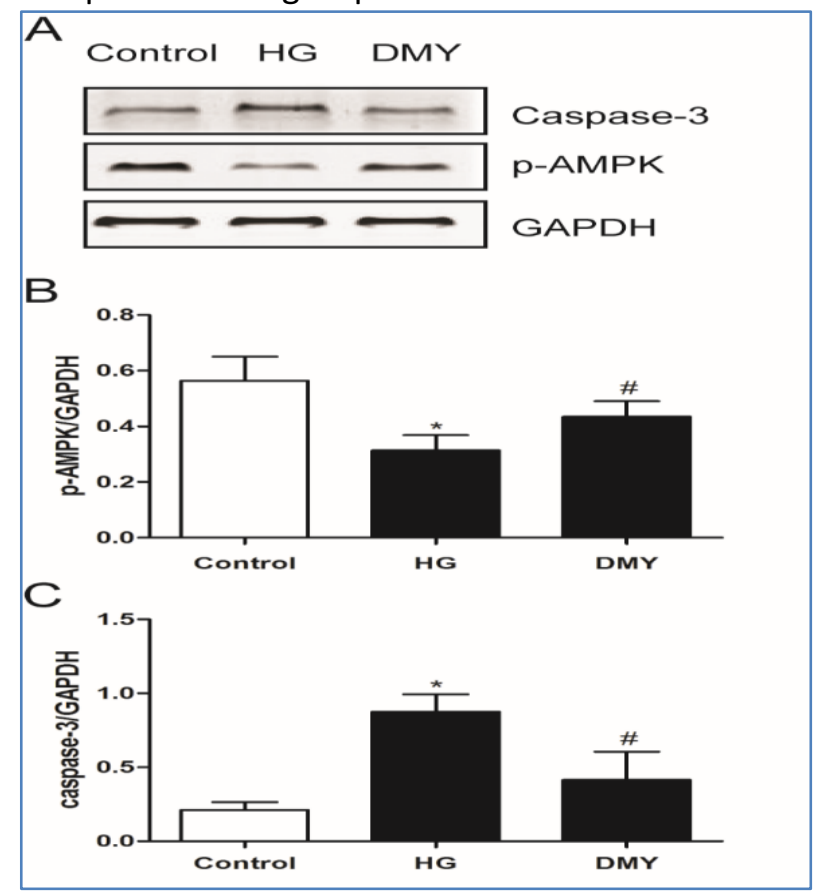

Fig.2: Effect of DMY on caspase-3 expression and AMPK phosphonylation in PC12 cells. PC12 cells are pretreated with DMY $20 \mu \mathrm{M}$ for $1 \mathrm{~h}$ and then exposed to HG for $48 \mathrm{~h}(\mathrm{~A})$. The density of caspase 3 and p-AMPK was determined by Western blotting and the ratio to GAPDH was calculated. ${ }^{*} P<0.05$ compared to control group, ${ }^{\#} P<0.05$ compared to HG group.

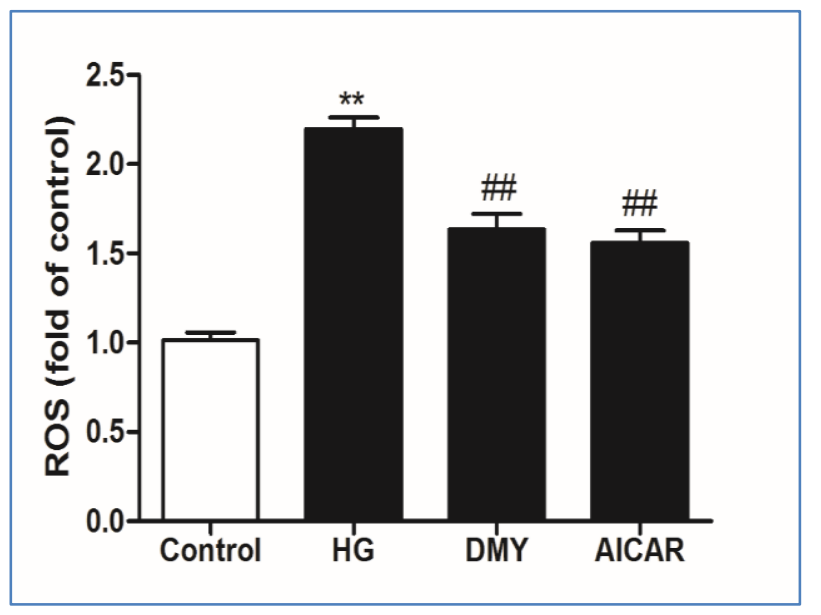

Fig.3: Effect of DMY and AMPK agonist on ROS production in PC12 cells. PC12 cells are pretreated with DMY $20 \mu \mathrm{M}$ or AMPK agonist AICAR $10 \mathrm{nM}$ for $1 \mathrm{~h}$ and then exposed to HG for $48 \mathrm{~h}$. Intracellular ROS generation in PC12 cells was determined using a detection assay. Values are expressed as ratio of control group and reported as mean $\pm \mathrm{SD} .{ }^{* *} P<$ 0.01 compared to control group. ${ }^{\# \#} P<0.01$ compared to HG group.

\section{Discussion}

In the present study, we reported that DMY pretreatment protected against HG-induced cell damage, and reduced the expression of caspase-3 and phosphorylated AMPK. DMY reduced ROS production possibly through AMPK pathway.

The flavonoids are a large a large class of powerful antioxidative compounds that are mainly found in fruits, tea and other plant-derived materials ${ }^{[11]}$. Many studies have demonstrated the effects of flavonoids and their metabolites on cancer, diabetes mellitus and Alzheimer's disease ${ }^{[12]}$. Dihydromyricetin has been showed a potential neuroprotective effect against oxidative stressinduced and amyloid-induced neuronal cell injury ${ }^{[13]}$. As a flavonoid compound, isolated from traditional southern Chinese herb Ampelopsis grossedentata, DMY possessed neuroprotective effects against oxidative stress in neurodegenerative diseases ${ }^{[14]}$. It was reported that DMY significantly reduced oxidative stress and inhibited microglial inflammation in transgenic mouse model of $A D^{[15]}$. Moreover, DMY also decreased oxidative stress induced cell injury in a concentration-dependent manner ${ }^{[16]}$. Consistent 
with previous studies, our results showed DMY protected PC12 neural cells against HG-induced cell cytotoxicity.

It's well known that imbalance of Bax/Bcl-2 leads to the release of cytochrome $C$, caspase- 3 activation and subsequent cell apoptosis in neural cells ${ }^{[17]}$. Caspase- 3 plays a pivotal role as a final stage of apoptosis ${ }^{[18]}$. It was reported that increased caspase-3 prompted apoptosis in PC12 cells ${ }^{[19]}$. Thus, our study found that DMY pretreatment is able to suppress the activation of capase-3 in PC12 cells.

Previous studies showed that hyperglycemiainduced intracellular ROS production and inhibition of AMPK phosphonylation ${ }^{[20]}$. We observed that the influences of DMY on inhibition of ROS production and activation of AMPK phosphonylation. Since AMPK signaling pathway is a crucial pathway involved in cell apoptosis. AMPK agonist also have similar protective effect with DMY treatment in HG-induced ROS level. It indicated that DMY might reduce cell damage and ROS production through AMPK pathway. Our study supported the AMPK signaling pathway involved in the HG-induced PC12 cell apoptosis and cytotoxicity.

In conclusion, our data highlight the activation of AMPK signaling pathway as a potential mechanism by which DMY protects PC12 cells from HG-induced ROS production and cell apoptosis. These results may suggest that DMY therapy could be employed as a promising agent in prevention therapy for diabetic neuropathy.

\section{Acknowledgement}

This work was partly supported by Science and Technology Development Program of Traditional Chinese Medicine in Shandong Province (2017207), Project of Shandong Province Higher Educational Science and Technology Program (J17KA256 and J18KA280), and Science and technology development project foundation of Weifang (2017YX063), Natural Science Foundation of Shandong Province (ZR2016HL12).

\section{References}

1. Semprini R, Martorana A, Ragonese M, Motta C. Observational clinical and nerve conduction study on effects of a nutraceutical combination on painful diabetic distal symmetric sensory-motor neuropathy in patients with diabetes type 1 and type 2.Minerva Med. 2018,109(5): 358-362.

2. Etienne I, Magalhães LVB, Cardoso SA, de Freitas RB, de Oliveira GP, Palotás A, Lima LM. Oxidative stress markers in cognitively intact patients with diabetic neuropathy. Brain Res Bull. 2019, 150: 196-200.

3. Sandireddy R, Yerra VG, Areti A, Komirishetty P, Kumar A. Neuroinflammation and oxidative stress in diabetic neuropathy: futuristic strategies based on these targets. Int J Endocrinol. 2014, 2014: 674987.

4. Huang B, Li Y, Yao Y, Shu W, Chen M. Dihydromyricetin from ampelopsis grossedentata protects against vascular neointimal formation via induction of TR3. Eur J Pharmacol. 2018, 838: 2331.

5. Zhang $X$, Li X, Fang J, Hou X, Fang H, Guo F, Li F, Chen $A$, Huang S. (2R,3R) Dihydromyricetin inhibits osteoclastogenesis and bone loss through scavenging LPS-induced oxidative stress and NF-KB and MAPKs pathways activating. J Cell Biochem. 2018, 119(11): 8981-8995.

6. Ling $H$, Zhu Z, Yang J, He J, Yang S, Wu D, Feng S, Liao D. Dihydromyricetin improves type 2 diabetesinduced cognitive impairment via suppressing oxidative stress and enhancing brain-derived neurotrophic factor-mediated neuroprotection in mice. Acta Biochim Biophys Sin (Shanghai). 2018, 50(3): 298-306.

7. Wang $Z$, Sun $X$, Feng $Y$, Liu $X$, Zhou L, Sui $H, J i ~ Q, E$ $Q$, Chen J, Wu L, Li $Q$. Dihydromyricetin reverses MRP2-mediated MDR and enhances anticancer activity induced by oxaliplatin in colorectal cancer cells. Anticancer Drugs. 2017, 28(3): 281-288

8. Qiu P, Dong Y, Li B, Kang XJ, Gu C, Zhu T, Luo YY, Pang MX, Du WF, Ge WH. Dihydromyricetin modulates p62 and autophagy crosstalk with the Keap-1/Nrf2 pathway to alleviate ethanol-induced hepatic injury. Toxicol Lett. 2017, 274: 31-41

9. Jiang $B$, Le L, Pan $H, H u K, X u ~ L$, Xiao $P$. Dihydromyricetin ameliorates the oxidative stress response induced by methylglyoxal via the AMPK/GLUT4 signaling pathway in PC12 cells. Brain Res Bull. 2014, 109: 117-26.

10. Sun $P$, Yin JB, Liu LH, Guo J, Wang $\mathrm{SH}$, Qu CH, Wang $\mathrm{CX}$. Protective role of Dihydromyricetin in Alzheimer's disease rat model associated with activating AMPK/SIRT1 signaling pathway. Biosci Rep. 2019, 39(1). pii: BSR20180902.

11. Zheng YZ, Deng G, Guo R, Fu ZM, Chen DF. The influence of the $\mathrm{H} 5$ ‥OC4 intramolecular hydrogenbond (IHB) on the antioxidative activity of flavonoid. Phytochemistry. 2019, 160: 19-24.

12. Bai $L$, Li $X$, He L, Zheng $Y$, Lu H, Li J, Zhong L, Tong R, Jiang Z, Shi J, Li J. Antidiabetic Potential of 
Flavonoids from Traditional Chinese Medicine: A Review. Am J Chin Med. 2019, 47(5): 933-957.

13. Jia L, Wang $Y$, Sang J, Cui W, Zhao W, Wei W, Chen $B$, Lu F, Liu F. Dihydromyricetin Inhibits $\alpha$-Synuclein Aggregation, Disrupts Preformed Fibrils, and Protects Neuronal Cells in Culture against AmyloidInduced Cytotoxicity. J Agric Food Chem. 2019, 67(14): 3946-3955.

14. Ren ZX, Zhao YF, Cao T, Zhen XC. Dihydromyricetin protects neurons in an MPTP-induced model of Parkinson's disease by suppressing glycogen synthase kinase-3 beta activity. Acta Pharmacol Sin. 2016, 37(10): 1315-1324.

15. Li C, Meng $P$, Zhang BZ, Kang $H$, Wen $H L$, Schluesener H, Cao ZW, Zhang ZY. Computer-aided identification of protein targets of four polyphenols in Alzheimer's disease (AD) and validation in a mouse AD model. J Biomed Res. 2019, 33(2): 101112.

16. Zhou DZ, Sun HY, Yue JQ, Peng Y, Chen YM, Zhong ZJ. Dihydromyricetin induces apoptosis and cytoprotective autophagy through ROS-NF-kB signalling in human melanoma cells. Free Radic Res. 2017, 51(5): 517-528.
17. Aminzadeh A. Protective effect of tropisetron on high glucose induced apoptosis and oxidative stress in PC12 cells: roles of JNK, P38 MAPKs, and mitochondria pathway. Metab Brain Dis. 2017, 32(3): 819-826.

18. Namazi Sarvestani $N$, Saberi Firouzi $S$, Falak $R$, Karimi MY, Davoodzadeh Gholami M, Rangbar A, Hosseini A. Phosphodiesterase 4 and 7 inhibitors produce protective effects against high glucoseinduced neurotoxicity in PC12 cells via modulation of the oxidative stress, apoptosis and inflammation pathways. Metab Brain Dis. 2018, 33(4): 12931306.

19. Veluthakal R, Arora DK, Goalstone $\mathrm{ML}$, et al. Metabolic stress induces caspase-3 mediated degradation and inactivation of farnesyl and geranylgeranyl transferase activities in pancreatic $\beta$-cells. Cell Physiol Biochem, 2016, 39(6): 21102120.

20. Peng $Y$, Liu J, Shi L, et al. Mitochondrial dysfunction precedes depression of AMPK/AKT signaling in insulin resistance induced by high glucose in primary cortical neurons. J Neurochem, 2016, 137(5): 701-713. 